\title{
Vaccine Safety and Pharmacovigilance in the African Region: Recent Updates
}

Bartholomew D Akanmori ${ }^{*}$, Tieble Traore ${ }^{1}$, M Balakrishnan $^{2}$, C Maure $^{2}$, P Zuber ${ }^{2}$, R Mihigo $^{2}$

IImmunization and Vaccines Development Programme, Family \& Reproductive Health Cluster, WHO Regional Office for Africa, Dioué, Brazzaville, Congo ${ }^{2}$ Safety and Vigilance, Essential Medicines and Health Products Department, Health Systems and Innovations Cluster, World Health Organization, 1211 Geneva 27, Switzerland

Article Info

\section{Article Notes}

Published: August 02, 2018

\section{${ }^{*}$ Correspondence:}

Bartholomew D. Akanmori, Immunization and Vaccines Development Programme, Family \& Reproductive Health Cluster, WHO Regional Office for Africa, Djoué, Brazzaville, Congo; Email: akanmorid@who.int

(C) 2018 Akanmori BD. This article is distributed under the terms of the Creative Commons Attribution 4.0 International License.

\section{Keywords:}

Vaccine safety

Pharmacovigilance

African Region

Immunization

AVAREF

\section{ABSTRACT}

Introduction: The number of subjects in clinical trials, is often limited and inadequate for detection of all adverse events which may be associated with vaccines, especially very rare ones. In addition, there is a surge in introduction of new vaccines into national immunization programmes in the WHO African Region, some of which have been used in a limited number of people, highlighting the need for functional national for pharmacovigilance systems for adverse events following immunization (AEFIs). Recognizing this, WHO and partners are supporting countries to develop national plans, providing training and investments in vaccine safety and pharmacovigilance. Despite these efforts, surveillance for vaccine safety in many countries remain weak. This paper reviews cases of $\mathrm{AEFI}$ reported by countries countries in the WHO/UNICEF Joint Reporting Form of WHO/AFRO between 2010 and 2015 , discusses some of the causes of the low reporting while exploring how countries can rely on new opportunities and systems to improve their reporting and vaccine safety in general.

Methodology: The implementation status of multi-stakeholder national plans developed by national immunization programmes, Pharmacovigilance Centres (PVCs) and the National Regulatory Authorities (NRAs) of 28 countries was reviewed. Using data from the WHO/UNICEF Joint Reporting Form and the introduction of new vaccines by countries in the WHO African, the impact of these plans on reporting of AEFIs was assessed for the countries.

Results: The analysis of performance revealed that only five countries have fully implemented plans for vaccine safety monitoring and pharmacovigilance in accordance with the Global Vaccine Safety Initiative (GVSI) blueprint. Implementation of the plans in the remaining 23 countries is slow. From 2010 - 2015, just 28 countries reported AEFIs as part of the WHO /UNICEF JRF. Yet $83 \%$ of countries introduced at least one new vaccine, with an average of 2 to 3 new vaccines being introduced per country over the period. Many countries have not fulfilled the responsibility of establishing expert committees on AEFI, developed guidelines, trained their staff on vaccine safety and put in place effective vaccine safety communication.

Discussion: The low AEFI reporting and weak pharmacovigilance demands special emphasis on capacity building, tailored to country needs to improve the reporting to meet the GVAP goals and UMC ADR guidelines. More sustainable support in ways that strengthen pharmacovigilance in general for all medical products and AEFI surveillance in particular in countries is needed. Opportunities are presented by the GVAP, the GVSI, networks such as the African Vaccine Regulatory Forum (AVAREF), Developing Countries Vaccine Regulatory Network (DCVRN), Developing Countries Vaccine Manufacturers Network (DCVM) and the International Federation of Pharmaceutical Manufacturers (IFPMA) as well the African Medicines Regulatory Harmonization (AMRH). African countries should exploit these opportunities to further strengthen their AEFI monitoring and pharmacovigilance.

\section{Introduction}

Vaccines are developed through carefully conducted series of studies in animals and humans, with the aim of establishing their 
efficacy or immunogenicity and safety. The data which are generated through clinical trials in humans are eventually submitted to regulatory authorities for the licensure and widespread use of the vaccines. Clinical trials have limited capacity to detect adverse events associated with vaccines, particularly very rare or late occurring events. The reasons are the relatively small number of subjects recruited for clinical trials, and the limit in follow-up times for participants restricts the probability of detection of events ${ }^{1-3}$. Clinical trials typically allow for the identification of untoward events with occurrence as rarely as 1 per 1,000 doses of vaccine administered. It is only after licensure when millions of people receive the vaccines, that very rare adverse events may be detected ${ }^{4}$. Furthermore, the existence of confounding factors (for example age, sensitization by related antigens, and concomitant infections) presents a difficulty for the investigation of rare, severe adverse reactions to vaccines during trials ${ }^{5}$. Moreover, health professionals and country regulators cannot rely entirely on the safety assessment conducted in developed countries for the novel or newly introduced vaccines since most will be launched exclusively or simultaneously in low and middle-income countries (LMICs). Evidence from GAVI, which funds most of the vaccines in use in the African Region shows that the interval between the launch of new vaccines in developed countries and their subsequent introduction in Africa has reduced considerably to months. This reduction in time means safety data from developed countries where the vaccines were first launched may be insufficient at the time of introduction in LMICs.

Post-market surveillance (PMS) thus ensures that each product is adequately monitored from its development to introduction into routine use. As a result, any rare or lateoccurring events can be identified, managed, recorded and communicated to all stakeholders and users ${ }^{6,7}$. Hence pharmacovigilance is a responsibility of end-users of the products, namely health professionals, manufacturers, regulators and the general public.

An adverse event by definition is any untoward medical occurrence associated with the use of a drug in humans, whether or not considered drug related. It may be a lifethreatening adverse event or a life-threatening suspected adverse reaction. The types of adverse events are well outlined in the WHO Global manual on surveillance of $\mathrm{AEFI}^{8}$. AEFIs are rare for all the currently licensed vaccines, But as the incidence of these vaccine preventable diseases decline, rare adverse events become more conspicuous, and benefitrisk ratio may appear to alter, particularly to a public which sees no disease against which the vaccine is protecting. If not well investigated and outcomes carefully communicated to a skeptical public, AEFIs, especially severe ones may lead to vaccine refusals and negatively affect immunization programmes in the region.

Reliable and sensitive surveillance systems for AEFI exist in many high-income countries but are lacking in the African
Region. Because of the rarity of severe reactions associated with routine vaccines, their surveillance often remains a low priority.

The Ebola virus disease (EVD) outbreak in the year 2014 necessitated accelerated research and development of new vaccines with several clinical trials spanning phases 1 to 3 , either completed or taking place across African countries, including the three worst affected countries ${ }^{9-11}$. The AEFIs associated with these vaccines are limited to the relatively small numbers of people exposed to these candidate vaccines in the clinical trials conducted so far, and to the duration of follow-up of each participant ${ }^{12-13}$.

Surveillance for AEFIs can be active, passive or stimulated, each with its own merits and limitations. The types of surveillance systems for AEFIs and their limitations are described in the literature ${ }^{14}$. Active surveillance involves intensive follow-up with examinations or questionnaires, sometimes including laboratory confirmation. Passive surveillance, on the other hand, depends on individual, unsolicited reporting by beneficiaries and ability to identify and willingness of health care providers to report AEFI. Passive surveillance is the most widely used system for PMS of vaccines. Stimulated surveillance is passive surveillance in which health workers are encouraged to report AEFIs ${ }^{15}$.

Passive surveillance often covers a large population which allows estimation of rates of rare events. AEFI rates cannot be accurately estimated by passive surveillance mainly because of the well-known under-reporting of this system. Passive surveillance is, however, useful to detect signals and to monitor general trends in reported AEFIs or to calculate reporting rates for particular AEFIs over time. Passive surveillance is also less expensive compared to active surveillance but provides rates of AEFIs, which are crude and may require special studies to confirm in some cases.

Active surveillance is dependent on active identification and reporting by health care providers and is resource intensive. This form of surveillance could be associated with many uncontrolled factors which can potentially affect the reporting rate, such as the level of interest or motivation of health workers and the threshold for the patient to seek medical attention. It may be difficult to determine the number of recipients vaccinated, i.e. the denominator.

Vaccines as biological products can vary from one batch or lot to the next, depending on the cultivation conditions of microorganisms as sources of their components. Therefore, one of the primary purposes of AEFI surveillance systems is to monitor vaccine lots routinely, to detect lots with an unusual number of associated adverse events. Events of infrequent occurrence caused by the vaccine are detected and estimates of rates of occurrence of rare illnesses following immunization established. The information can then be used to make a comparison of rates by type of vaccine, to raise awareness of health workers of risks of vaccines, and to identify areas that require special investigation. 
However, the burden is to provide adequate and timely evidence of the safety of these new vaccines in wider use, through appropriate surveillance systems, to identify and manage any risks, provide responses to decision-makers as well as the general public, to maintain trust in vaccines and immunization. Within this context the WHO Regional Office for Africa (WHO-AFRO) developed a strategy to strengthen vaccine safety and pharmacovigilance in its Member States.

\section{Methodology}

Data was collected from countries by WHO on the new vaccines which were introduced over the past five years and tabulated. We also reviewed the data contained in the WHO/UNICEF Joint Reporting Form, which is obtained annually from countries and includes the number of cases of AEFIs reported by countries. In 2014, 24 African countries developed national guidelines for monitoring AEFIs in line with the updates and recommendations of WHO with the recommended core variables for AEFI reporting and standardized investigation and causality assessment processes. All the data was analyzed and formed the basis of the results which are presented in this manuscript.

Firstly, review of the number of countries reported to have introduced new vaccines, together with the number of cases of AEFIs. Secondly, the status of implementation of national plans for pharmacovigilance revealed the specific areas requiring attention in order to address the low reporting. These results are presented in tables and discussed. Thirdly associations between low reporting of AEFIs and status of implementation of national plans was carried out. Finally, the opportunities for addressing the weak pharmacovigilance was discussed, with clearly defined support systems available to countries duly highlighted.

\section{Results}

\section{Availability of additional vaccines in the past five years}

The African Region which has seen an unprecedented surge in introductions of additional vaccines into immunization programmes, averaging 2 to 3 per country over the last 5 years (Table 1 ). However, the corresponding increase in reported AEFIs has only been modest (2010 896 cases, 2011 -513 cases, 2012 - 2244 cases, 2013 - 3712 cases and $2014-14,627$ cases). In 2014, Burkina Faso alone contributed 9,539 (65\%) of the total number of AEFIs in the region (Table 2). The evidence from the World Health Organization (WHO) and United Nations Children's Fund (UNICEF) Joint Reporting Form, therefore, points to very low reporting rate for AEFIs in the region, despite the increase in the number of vaccines in use. Table 1 shows countries and the vaccines they have introduced into their National Immunization Programmes (NIPs) within from 2010 to 2015. In the period, 2010 and 2015, 39 (83\%) out of 47 countries of the WHO African Region introduced at least one additional vaccine into their routine immunization programme. Six of the countries introduced at least three or more vaccines. In
Table 1: Vaccines introduced in past five years (2010-2015) in the countries of the WHO African Region

\begin{tabular}{|c|c|c|c|}
\hline \multirow[t]{2}{*}{ Country } & \multicolumn{3}{|c|}{$\begin{array}{c}\text { Additional Vaccines Introduced past } 5 \\
\text { years }\end{array}$} \\
\hline & 1 & 2 & $\geq 3$ \\
\hline Angola & & Rota, Pneumo & \\
\hline Benin & Pneumo & & \\
\hline Botswana & & & $\begin{array}{c}\text { Rota, Pneumo, } \\
\text { HPV }\end{array}$ \\
\hline Burkina Faso & & & $\begin{array}{c}\text { Rota, Pneumo, } \\
\text { MenA }\end{array}$ \\
\hline Burundi & & Rota, Pneumo & \\
\hline Cameroun & & Rota, Pneumo & \\
\hline Central African Republic & Pneumo & & \\
\hline Congo & & Rota, Pneumo & \\
\hline Côte d'Ivoire & Pneumo & & \\
\hline $\begin{array}{l}\text { Democratic Republic of } \\
\text { Congo }\end{array}$ & Pneumo & & \\
\hline Eritrea & & Rota, Pneumo & \\
\hline Ethiopia & & Rota, Pneumo & \\
\hline Gambia & Rota & & \\
\hline Ghana & & & $\begin{array}{c}\text { PCV, Rota, HPV } \\
\text { MenA }\end{array}$ \\
\hline Guinea-Bissau & & Rota, Pneumo & \\
\hline Kenya & & Rota, Pneumo & \\
\hline Lesotho & & Pneumo, HPV & \\
\hline Liberia & Pneumo & & \\
\hline Madagascar & & Rota, Pneumo & \\
\hline Malawi & & Rota, Pneumo & \\
\hline Mali & & & $\begin{array}{c}\text { Rota, Pneumo, } \\
\text { MenA }\end{array}$ \\
\hline Mauritania & & Rota, Pneumo & \\
\hline Mauritius & Rota & & \\
\hline Mozambique & & Rota, Pneumo & \\
\hline Namibia & & Rota, Pneumo & \\
\hline Niger & & & $\begin{array}{c}\text { Rota, Pneumo, } \\
\text { MenA }\end{array}$ \\
\hline Nigeria & & $\begin{array}{l}\text { Pneumo, } \\
\text { MenA }\end{array}$ & \\
\hline Rwanda & & Rota, HPV & \\
\hline Sao Tome \& Principe & Pneumo & & \\
\hline Senegal & & & $\begin{array}{c}\text { Rota, Pneumo, } \\
\text { MenA }\end{array}$ \\
\hline Seychelles & HPV & & \\
\hline Sierra Leone & & Rota, Pneumo & \\
\hline South Africa & HPV & & \\
\hline Swaziland & & Rota, Pneumo & \\
\hline Togo & & Rota, Pneumo & \\
\hline Uganda & Pneumo & & \\
\hline $\begin{array}{l}\text { United Republic of } \\
\text { Tanzania }\end{array}$ & & Rota, Pneumo & \\
\hline Zambia & & Rota, Pneumo & \\
\hline Zimbabwe & & Rota, Pneumo & \\
\hline
\end{tabular}


summary, 28 (60\%) countries have introduced two or more new vaccines into their immunization programmes. Despite, this increase in vaccine introduction, there has not been a corresponding rise in AEFIs and reporting is not consistent (Table 2) and therefore vaccine pharmacovigilance continues to present a challenge.

Detailed analysis reveals that in the period 2010 to 2014, just 28 countries reported AEFIs as part of the WHO /UNICEF Joint Reporting Form. The reporting is not consistent, with between 16 and 19 countries failing to report any AEFIs from 2010 to 2014, out of an expected total of 46 countries (2010 to 2013) and 47 in 2014. Also, the countries which reported AEFIs in the period did not meet the expected reporting rates annually as per table above. For example, Nigeria reported just 3864 compared to 11755 cases reported by Burkina Faso with an estimated population which is just a fraction of that of Nigeria (Table 2). This silence in reporting or low reporting may perhaps reflect the absence of robust AEFI monitoring systems, lack of guidelines and AEFI review committees, unavailability of trained personnel and weak collaboration among stakeholders, despite the inclusion of AEFIs in the national Integrated Disease Surveillance and Response (IDSR) systems.

\section{Opportunities for strengthening vaccine safety and pharmacovigilance}

The WHO Global Vaccine Action Plan (GVAP): The GVAP is a global framework for immunization, endorsed by all countries. It has six clearly stated strategic objectives which also served as the guiding principles for the development of the plan. These guiding principles underpin the vision of a decade of vaccines (2011-2020), with an ideal of a world in which all individuals and communities enjoy lives free from vaccine-preventable diseases. The 4 th of the six strategic objectives of the GVAP states that strong immunization systems are built as an integral part of a wellfunctioning health system. To attain this objective, the GVAP calls for ensuring that everyone everywhere receives the safest vaccines possible and that safety concerns are not a cause of hesitancy in using vaccines. Implementation of the GVAP therefore presents an opportunity for countries to develop plans for immunization comprehensively, including monitoring and reporting of all AEFIs and to contribute to strengthening pharmacovigilance.

The Global Vaccine Safety Blueprint (GVSB) and Global Vaccine Safety Initiative (GVSI): Reliable framework and enduring initiative: To support the LMICs to establish and strengthen vaccine safety monitoring systems, WHO launched the Global Vaccine Safety Blueprint (GVSB). The GVSB is the basis for countries to plan and implement vaccine safety activities. These safety activities include monitoring, reporting, investigating and disseminating information about vaccine adverse events ${ }^{16}$. The GVSB is accompanied by a global portfolio of vaccine
Table 2: List of countries in the WHO AFR which reported at least one case of AEFI in the period 2010 to 2014

\begin{tabular}{|c|c|}
\hline Country & All AEFIs reported \\
\hline Algeria & 471 \\
\hline Burkina Faso & 11755 \\
\hline Cabo Verde & 2 \\
\hline Cameroon & 2244 \\
\hline Central African Republic (the) & 52 \\
\hline Comoros (the) & 11 \\
\hline Côte d'Ivoire & 1745 \\
\hline Eritrea & 11 \\
\hline Ethiopia & 18 \\
\hline Gambia (the) & 14 \\
\hline Ghana & 31 \\
\hline Kenya & 23 \\
\hline Liberia & 1 \\
\hline Malawi & 3 \\
\hline Mauritania & 35 \\
\hline Mauritius & 7 \\
\hline Namibia & 3 \\
\hline Nigeria & 3854 \\
\hline Rwanda & 374 \\
\hline Sao Tome and Principe & 10 \\
\hline Senegal & 143 \\
\hline Sierra Leone & 27 \\
\hline South Africa & 705 \\
\hline Swaziland & 5 \\
\hline Togo & 227 \\
\hline Uganda & 46 \\
\hline United Republic of Tanzania & 214 \\
\hline Zimbabwe & 270 \\
\hline
\end{tabular}

Data was unavailable for Algeria, Cape Verde, Chad, Comoros, Equatorial Guinea, Gabon, Guinea and South Sudan.

safety activities being undertaken. Also, the WHO created a technical support network, the Global Vaccine Safety Initiative (GVSI) comprising partners and experts to support technical capacity building in countries (World Health Organization (8). The GVSB and GVSI have provided a strong framework for the capacity building in the African Region. It remains the backbone of vaccine safety activities in the region.

\section{Other opportunities for strengthening vaccine safety and pharmacovigilance}

The establishment of functional systems for vaccine safety and pharmacovigilance requires resources, financial, human and other partner inputs. The current environment offers some opportunities to countries in the region. Vaccine safety and pharmacovigilance is receiving attention from many stakeholders. New tools and guidelines have been published for evaluation of new types of vaccines and technologies ${ }^{17,18}$.

The Bill and Melinda Gates Foundation and the GAVI 
Alliance have prioritized this area of work and set up vaccine safety and pharmacovigilance teams. Opportunities exist for capacity building, harmonization, sharing of best practices and mentorships in support of pharmacovigilance, through a variety of networks. The biennial GVSI meetings offer opportunity for networking, sharing experiences and best practices. The African Medicines Regulatory Harmonization Initiative (AMRHI), the African Vaccine Regulatory Forum (AVAREF) and the Developing Countries Vaccine Regulatory Network (DCVRN), Developing Countries Vaccine Manufacturers Network (DCVM) and the International Federation of Pharmaceutical Manufacturers (IFPMA) represent additional platforms for networking.

\section{Requirements and Institutional arrangements for monitoring AEFIs}

Every country which utilizes medical products is required to have a reliable system in place for monitoring the safety of all products, including vaccines, throughout their lifecycles. The WHO through the GVSB has identified some minimum requirements for every country to monitor AEFIs effectively.

Each country should also consistently report AEFIs as part of the data collected through the WHO/UNICEF Joint Reporting Form (JRF) as required by the GVAP. In the rare cases of severe AEFIs, the country should have in place an expert committee capable of investigating and ultimately carrying out causality assessment, to establish the real cause of the adverse event and respond to it based on the findings of the assessment.

To achieve this, each country should ensure the involvement of the appropriately mandated institutions, namely, the National Regulatory Authority (NRA), the National Immunization Programme or EPI, Disease Surveillance Unit of the Ministry of Health and a centre for pharmacovigilance, if in place in monitoring AEFIs. All the institutions and other stakeholders including WHO and UNICEF, working in harmony, but with clearly defined roles and responsibilities will ensure that the vaccine safety and pharmacovigilance system functions very well. The NIP, which carries out all immunizations through various health outlets routinely and periodic campaigns, should have the primary responsibility for collecting reports of all AEFIs.

Secondly, a clear data and information flow pathway should be identified, so that AEFIs are communicated quickly to the NRAs which licensed the vaccines, manufacturers, as well as the pharmacovigilance centres whose mandate covers all medical products. Thus, all events once collected should be accessible to the pharmacovigilance system and NRA at a pre-defined level of the local government, district, regional or national. These events should be reviewed, entered into a national database, analyzed and comparisons made with data obtained from elsewhere for the same products. The desirable flow of information and reports is displayed in Figure 1. Although applicable to all countries, the flowchart may vary from country to country, depending on institutional mandates, roles and responsibilities and the levels of health delivery. Typically, information on adverse events is systematically collected using the WHO standard forms at immunization points, health facility, district hospital, regional hospital or during campaigns. All notified cases should have a reporting form with basic core information, matching the 25 core variables in the WHO AEFI Guidelines, completed ideally by the first health care provider. If the case is recognized as serious, a detailed investigation is required to determine the underlying cause. All reported cases should be line-listed and the data analyzed to determine signals.

\section{Integrated Disease Surveillance and Response}

The Integrated Disease Surveillance and Response (IDSR) is a strategy for developing and implementing a comprehensive national public health surveillance and response system, currently used in African countries. In June 2010, results from a survey indicated that 45 countries of the WHO/AFR had identified IDSR priority diseases or conditions.

The IDSR, therefore, offers an excellent opportunity to strengthen the AEFI surveillance. In the IDSR Technical Guidelines (2010), AEFI is listed among the priority diseases, conditions and events of public health importance requiring immediate notification. Furthermore, questions related to recent vaccination history are an integral part of the outbreak investigation, particularly during the initial phase of reviewing the clinical history and epidemiology of outbreaks. Examples of key signs and symptoms of AEFI case definitions are described in the IDSR guide. Practically, surveillance activities for different diseases involve similar functions (detection, reporting, analysis and interpretation, feedback, action) and more importantly use the same structures, processes, and personnel as shown in the flow chart.

\section{The National Regulatory Authority}

The National Regulatory Authority (NRA), an institution, which is legally mandated to ensure that products, typically pharmaceuticals and biologicals, sometimes food and cosmetics as well, are duly evaluated for their safety and quality to make sure these meet international standards, before marketed for use by the public, is fundamental to monitoring AEFIs. Recognizing this, the Expert Committee on Biological Standardization of WHO recommended that each country should a have a functional NRA to monitor vaccines for the quality and safety of vaccines and to ensure that these meet international standards. With the support of WHO and partners, countries made the efforts to establish NRAs and to capacity over the years for all regulatory functions including post-market surveillance and pharmacovigilance ${ }^{18}$. Countries of the African region were supported to develop institutional development plans and are periodically assessed for the performance of their regulatory functions. 
Personnel- Dedicated, national vaccine pharmacovigilance capacity, with designated staff for the purpose of collecting, analysis, reporting and communicating about vaccine safety;

Funding- Stable basic funding, clear mandates, well defined structures and roles

Collaboration- Effective collaboration with the WHO Programme for International Drug Monitoring

Reporting- Health-care workers and others who are encouraged to report vaccine safety issues

Reporting forms- Reporting form for individual case safety reports (i.e. a national reporting form for AEFI)

Database- National database or system for collating, managing and retrieving AEFI reports;

AEFI Committee- National AEFI expert review committee (ARC) able to provide technical assistance on causality assessment of serious AEFI and clusters of AEFI so that unwanted risk can be managed

Tools and methods - Harmonized methods and tools for the monitoring and investigation of AEFI

Risk management strategy- Strategy for risk communication that identifies and communicates risks and benefits to all (health professionals, caregivers, and public)

Figure 1: Key requirements and institutional arrangements for monitoring AEFIs

A survey of regulators was conducted in 2010, to identify the obstacles to the implementation of vaccine safety systems. Among the factors identified are lack of standardized AEFI reporting forms, no reliable systems for surveillance, inadequate expertise and training, no exchange and access to safety data as well as low national commitment and enforcement ${ }^{19}$. Since then countries have been supported to address these challenges through the WHO Global Vaccine Safety Blueprint ${ }^{20}$.

All countries are therefore now required to have an NRA, which meets the key functions, including systems for vaccine safety and pharmacovigilance. All the 47 countries have NRAs which are at different levels of performance and are carrying out some of the functions outlined.

\section{The National Immunization Programme}

Although vaccines like other medicines are licensed by NRAs, they are administered to populations either routinely or through campaigns by national immunization programmes, the EPI. The immunization programme is therefore also responsible for the collection of AEFIs, collation, and transmission to the NRA and pharmacovigilance centres where they exist. The NIPs of most countries routinely collect AEFI data as part of their activities in immunization. However, not much attention is paid to the AEFI reports unless they are severe. Some countries report AEFIs as part of the WHO/UNICEF Joint Reporting Form, while others do not. The data is rarely analyzed and communicated to other health professionals and the general public. The NIP has a responsibility to collect AEFI data and to share it with all the stakeholders (Figure 2).

\section{National Centres for pharmacovigilance}

These are national pharmacovigilance centres (PVC) which are recognized by WHO and are participating in the WHO Programme for International Drug Monitoring. The centres are either independent, part of or linked to NRAs. These centres regularly receive individual case safety reports (ICSRs) from healthcare professionals and patients and forward them to the central WHO Global ICSR database, which is called VigiBase, that is managed and maintained by the WHO collaborating centre - Uppsala Monitoring Centre Sweden. The events which are reported by the PVCs include AEFIs, even though these are fewer in some cases than that of drug reactions. To ensure that AEFIs are also captured countries must ensure that the have PVCs and that these receive AEFI data from the EPI or NRA for onward transmission to the global database at the UMC.

\section{National Integrated Plans}

The primary step is to establish a system for monitoring AEFIs which involves all the stakeholders, ensuring that AEFI's data are collected routinely, analyzed and used in the country. To ensure continued or improved reporting of AEFIs and sharing of data with the PVC, NRA and the WHO Programme for International Drug Monitoring, WHO conducted multi-stakeholder workshops for 


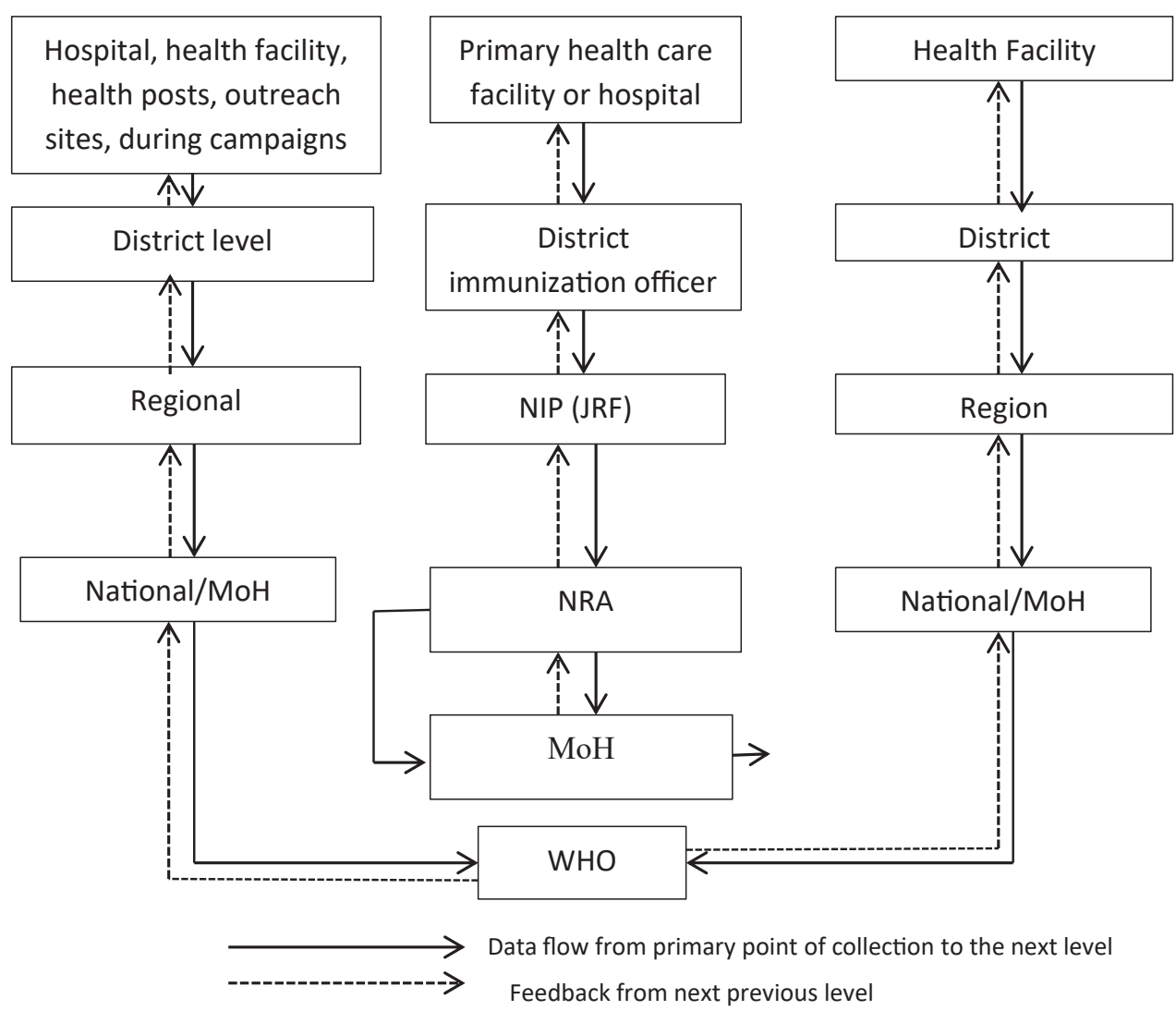

Figure 2: Simplified flow chart showing information flow of EPI, pharmacovigilance and integrated disease surveillance data (Adapted from WHO IDSR Guide and WHO AEFI Guidelines)

the development of national plans. The focus of these planning workshops was on improving reporting from the periphery of the health care system to the national level, use of standardized procedures and to operationalize the institutional development plans (IDPs) of the NRAs. The workshops involved NRA, EPI, PVC disease surveillance focal points in some cases and with the support of WHO Country staff responsible for Essential Medicines and Products and EPI. The national plans which are consistent with National Health Strategic Plans fulfilled clear requirements, which are illustrated in Table 3.

Countries prioritized their activities and developed a timeframe for the activities outlined in the work plan developed.
In a series of four workshops for groups for Frenchspeaking and English-speaking countries held in 2014 and 2015, 28 countries developed plans and are implementing them starting with three main priorities of these plans. Table 4 shows the countries, their priority activities identified and the status of implementation by date 2014-2016. The priorities identified are the initiation of meetings between the NRA, EPI, PVC and other stakeholders to establish and maintain vaccines safety and pharmacovigilance, the establishment of National AEFI Expert committees, identification of training needs and conducting at least one course or workshop for training of trainers, with or without simulations. Simulations are practical exercises in response to a reported adverse event in a health facility

Table 3. Charateristcs of national intergrated plans for vaccine safety and pharmacovigilance

\begin{tabular}{|c|c|}
\hline Aspect & Charectristic \\
\hline Process of Development & Developed with inputs from all stakeholders - NRA, EPI, and PVC \\
\hline Scope & Identifies clear roles and responsibilities \\
\hline Components & $\begin{array}{l}\text { - Identifies the establishment of independent AEFI Expert Committee or as part of existing pharmacovigilance } \\
\text { expert committees } \\
\text { - Development of national guidelines for AEFI monitoring and response } \\
\text { - Development of charts, posters and other aids and tools for health workers } \\
\text { - Training plans for committees of experts and other categories of health workers } \\
\text { Three key priories identified and budgeted for immediate implementation }\end{array}$ \\
\hline
\end{tabular}


Table 4. Priority activities out of four identified which have been implemented by countries.

\begin{tabular}{|c|c|c|c|c|}
\hline Country & $\begin{array}{l}\text { Meetings between } \\
\text { NRA, EPI, and other } \\
\text { stakeholders }\end{array}$ & $\begin{array}{l}\text { Constitution of National } \\
\text { AEFI expert committees }\end{array}$ & $\begin{array}{c}\text { Training of expert } \\
\text { committees in causality } \\
\text { assessment }\end{array}$ & $\begin{array}{l}\text { Simulations of } \\
\text { investigations }\end{array}$ \\
\hline \multicolumn{5}{|l|}{ Côte d'Ivoire } \\
\hline \multicolumn{5}{|l|}{ Rwanda } \\
\hline Burundi & Yes & & No & \\
\hline \multicolumn{5}{|l|}{ Niger } \\
\hline \multicolumn{5}{|l|}{ Mauritania } \\
\hline \multicolumn{5}{|l|}{ Mali } \\
\hline \multicolumn{5}{|l|}{ Malawi } \\
\hline Cameroun & Yes & No & No & \\
\hline Madagascar & Yes & No & No & \\
\hline Democratic Republic of Congo & Yes & No & No & \\
\hline Ethiopia & Yes & Yes & Yes & \\
\hline Nigeria & Yes & Yes & No & \\
\hline Uganda & Yes & Yes & No & \\
\hline Kenya & Yes & No & No & \\
\hline Mauritania & Yes & No & No & \\
\hline Benin & Yes & No & No & \\
\hline Togo & Yes & No & No & \\
\hline Ghana & Yes & Yes & No & \\
\hline Senegal & Yes & Yes & No & \\
\hline Burkina Faso & Yes & Yes & Yes & \\
\hline S Sudan & Yes & No & No & \\
\hline Namibia & Yes & Yes & No & \\
\hline Lesotho & Yes & No & Training & \\
\hline Eritrea & Yes & Yes & No & \\
\hline The Gambia & Yes & Yes & No & \\
\hline S Leone & Yes & No & No & \\
\hline Liberia & Yes & No & No & \\
\hline Guinea & Yes & Yes & Yes & \\
\hline Tanzania & Yes & Yes & Yes & Yes \\
\hline Zambia & Yes & Yes & Yes & \\
\hline Zimbabwe & Yes & Yes & Yes & Yes \\
\hline Total & 25 & 24 & 25 & 2 \\
\hline
\end{tabular}

with health workers responding based on the training they have received. This exercise has been conducted in Tanzania and found to be very useful in building capacity. The status of implementation, as at December 2015 varies from 5 countries (18\%) which have fulfilled all the priorities, to 15 (89\%) which fulfilled two and to 17 (64\%) which have carried out just one task. In teleconferences, countries highlighted the lack of technical capacity to conduct the training of experts, administrative requirements for initiation of intersectoral collaboration, competing activities as main reasons for delays in implementation.

\section{Priority activities implemented so far}

Since 2010, the African region has experienced a significant increase in availability of additional vaccines for national immunization programmes, than any other period since the establishment of the Expanded Programme on
Immunization (EPI) in $1974^{15}$. The countries have already begun meetings involving all the stakeholders in vaccine safety and pharmacovigilance, namely the NRA, EPI, PC, WHO, UNICEF and other stakeholders. Information is now shared and planning, and implementation involves all parties. Some countries have established or reactivated their AEFI expert committees, which meet to review AEFI data. AEFI committees in Tanzania, Zimbabwe, Zambia, and Ethiopia, have undergone training in vaccine safety and pharmacovigilance, including simulation of a response to a severe adverse event in Tanzania. In these countries, the core of AEFI expert committee members who were trained will serve as trainers for subsequent in-country training at regional and district levels.

\section{Future perspectives}

The future of vaccine safety and pharmacovigilance in 
Africa looks bright. The first steps in the entire process of ensuring that countries build robust vaccine systems for vaccine safety and pharmacovigilance have been put in place. The planning workshops were successful, and the participating countries have developed national plans which are consistent with their national health strategic plans and are implementing of them. Adequate, resources will have to be mobilized, especially from internal sources, adequate capacity built, training plans developed, and new policy changes effected.

WHO has focused on training a set of potential trainers and creating a network of trainers who could cascade the training activities down to the lowest levels. This roster of trainers will be kept updated and used to drive vaccine safety training in the region.

The GVAP and UMC ADR reports will be used to monitor the progress of countries, with quarterly reporting, to ensure that they remain on track.

\section{Conclusion}

While the implementation of national plans for vaccine safety and pharmacovigilance is slow in countries of the region opportunities exist for further strengthening the capacities of countries, through various networks and platforms. The GVSB is an excellent framework for development of national plans, priority training and courses were undertaken to improve reporting of AEFIs across countries of the region, as they make progress towards the status of better resourced developed countries ${ }^{19}$. Much more needs to be done in African countries, exploiting innovative means and taking advantage of current opportunities for them to meet the basic requirements for reliable monitoring of AEFIs.

\section{Conflict of Interest}

None

\section{References}

1. Miller ER, Haber P, Hibbs P, et al. Surveillance for Adverse Events Following Immunization Using the Vaccine Adverse Event Reporting System (VAERS). Manual for the Surveillance of Vaccine-Preventable Diseases: Centers for Disease Control and Prevention (CDC). 2011; $1-13$.

2. Autran B, Asturias EJ, Evans S, et al. Global safety of vaccines: strengthening systems for monitoring, management and the role of GACVS. Expert Rev Vaccines. 2009; 8: 705-16.

3. Ellenberg SS, Foulkes MA, Midthun K, et al. Evaluating the safety of new vaccines: summary of a workshop. Am J Public Health. 2005; 95 : 800-7.
4. Clemens J, Brenner R, Rao M, et al. Evaluating new vaccines for developing countries: efficacy or effectiveness. Jama. 1996; 275: 390 7.

5. Fine PE, Chen RT. Confounding in studies of adverse reactions to vaccines. Am J Epidemiol. 1992; 136: 121-35.

6. Miller ER, Moro PL, Cano M, et al. Post-licensure safety surveillance of 23-valent pneumococcal polysaccharide vaccine in the Vaccine Adverse Event Reporting System (VAERS), 1990-2013. Vaccine. 2016; 34(25): 2841-6.

7. Salmon DA, Pavia A, Gellin B. Editors' introduction: Vaccine safety throughout the product life cycle. Pediatrics. 2011;127 Suppl 1: S1-4.

8. World Health Organization. Global manual on surveillance of severe adverse events following immunization. 2016. http://www.who. int/vaccine_safety/publications/Global_Manual_revised_12102015. pdf?ua $=1$

9. Rampling T, Ewer K, Bowyer G, et al. A Monovalent Chimpanzee Adenovirus Ebola Vaccine - Preliminary Report. N Engl J Med. 2015.

10. Henao-Restrepo AM, Longini IM, Egger M, et al. Efficacy and effectiveness of an rVSV-vectored vaccine expressing Ebola surface glycoprotein: interim results from the Guinea ring vaccination clusterrandomised trial. Lancet. 2015; 386: 857-66.

11. Agnandji ST, Huttner A, Zinser ME, et al. Phase 1 Trials of rVSV Ebola Vaccine in Africa and Europe - Preliminary Report. N Engl J Med. 2015; 374(17): 1647-60.

12. The European Medicines Agency (EMA). First malaria vaccine receives a positive scientific opinion from EMA. 2015.

13. World Health Organization (WHO). The Weekly Epidemiological Record (WER). Weekly Epidemiological Record (WER) 2016; 33-52.

14. Crawford NW, Clothier H, Hodgson K, et al. Active surveillance for adverse events following immunization. Expert Rev Vaccines. 2014; 13: 265-76.

15. Okwo-Bele JM, Cherian T. The expanded programme on immunization: a lasting legacy of smallpox eradication. Vaccine. 2011; 29 Suppl 4: D74-9.

16. World Health Organization. WHO Blueprint for Vaccine safety. The Global Vaccine Safety Initiative (GVSI) WHO. Geneva 2012.

17. U.S. Food and Drug Administration (FDA). 21 CFR Part 4 [Docket No. FDA-2009-N-0435], Current Good Manufacturing Practice Requirements for Combination Products. In: SERVICES DOHAH, editor. 2013; 4307-23.

18. Committee for Medicinal Product for Human Use (CHMP). Guideline on quality, non-clinical and clinical aspects of love recombinant viral vectored vaccines. In: Agency EM, editor. EMA/CHMP/ VWP/141697/20092010.

19. Graham JE, Borda-Rodriguez A, Huzair F, et al. Capacity for a global vaccine safety system: The perspective of national regulatory authorities. Vaccine. 2012; 30: 4953-9.

20. Chen RT, Shimabukuro TT, Martin DB, et al. Enhancing vaccine safety capacity globally: A lifecycle perspective. Vaccine. 2015; 33, Supplement 4: D46-D54. 